\title{
Donantes de órganos en muerte cerebral por herida por proyectil de arma de fuego en cráneo: una trágica realidad que beneficia receptores de órganos en Colombia
}

\author{
Fire arm wounded cerebral death organ donors: a tragic reality that benefits \\ organ receptors in Colombia
}

\author{
Ana M. Aristizábal1,2, Catalina Gómez-Duarte1,2, Víctor H. García1,2, Eliana Manzi³, \\ Carlos Escobar ${ }^{4}$, Juan David Jaramillo4, Karen Torres', Mauricio Millán ${ }^{6}$, Luis A. Caicedo6, \\ Gabriel J. Echeverri ${ }^{7}$ \\ 1 Médico general, investigador, Centro de Investigaciones Clínicas, Fundación Valle del Lili, Cali, Colombia \\ 2 Médico general, investigador, Centro para la Investigación en Cirugía Avanzada y Trasplantes (CICAT), Universidad ICESI, Cali, Colombia \\ 3 Especialista en Estadística, Centro de Investigaciones Clínicas, Fundación Valle del Lili, Cali, Colombia \\ 4 Estudiante de Medicina, Facultad de Ciencias de la Salud, Universidad ICESI, Cali, Colombia \\ 5 Coordinador operativo de trasplantes, Departamento de Trasplantes, Centro de Investigaciones Clínicas, Fundación Valle del Lili; \\ Centro para la Investigación en Cirugía Avanzada y Trasplantes (CICAT), Universidad ICESI, Cali, Colombia \\ 6 Especialista en Cirugía de Trasplante de Órganos Abdominales, Departamento de Trasplantes, Centro de Investigaciones Clínicas, \\ Fundación Valle del Lili; Centro para la Investigación en Cirugía Avanzada y Trasplantes (CICAT), Universidad ICESI, Cali, Colombia \\ 7 Especialista en cirugía de trasplante de órganos abdominales, Colombiana de Trasplantes, Barranquilla, Colombia
}

\section{Resumen}

Antecedentes y objetivo. Los pacientes con muerte encefálica por trauma craneoencefálico secundario a herida por proyectil de arma de fuego ayudan a mitigar la necesidad de donantes de órganos y tejidos. En Colombia, el $78 \%$ de los homicidios son por arma de fuego. Se buscaba describir la experiencia con donantes de órganos y tejidos con muerte encefálica secundaria a trauma craneoencefálico por herida por proyectil de arma de fuego en un centro colombiano de trasplantes.

Materiales y métodos. Se trató de un estudio de cohorte histórica retrospectiva. Se caracterizaron I69 donantes con diagnóstico de muerte encefálica secundaria a trauma craneoencefálico por herida con proyectil de arma de fuego en la institución, entre 20I0 y 2016. Las variables cualitativas se evaluaron mediante proporciones, las variables cuantitativas continuas, con medidas de tendencia central, y la supervivencia, con el método de Kaplan-Meier.

Resultados. La mayoría de los pacientes eran de sexo masculino (93\%), con una mediana de edad de 2I años (rango intercuartílico, RIQ=I7-27) y el $32 \%$ tenía antecedentes de consumo de sustancias psicoactivas. El I2 \% de los pacientes necesitó manejo con hemoderivados y el 62 \% requirió dosis altas de vasopresores. Se trasplantaron 338 órganos sólidos. La supervivencia del injerto renal y hepático a un año fue de $90 \%$ y $85 \%$, respectivamente, y a 5 años, de $87 \%$ y $76 \%$.

Conclusiones. Según este estudio, la mayoría de estos pacientes eran hombres jóvenes con órganos potencialmente trasplantables que mostraron excelentes resultados en la supervivencia de los injertos. Consideramos que estos donantes son una buena alternativa para aumentar el número de donantes de órganos y tejidos.

Palabras clave: trasplante de órganos; selección de donante; muerte encefálica; traumatismos cerebrovasculares; lesiones traumáticas del encéfalo; traumatismos penetrantes de la cabeza.

Fecha de recibido: 1/12/2017 - Fecha de aceptación: 24/01/2018

Correspondencia: Gabriel J. Echeverri, Cirugía de Trasplantes, Colombiana de Trasplantes, Barranquilla, Colombia. Teléfono: (318) 287-9637 / Correo electrónico: gjecheverri@hotmail.com

Citar como: Aristizábal AM, Gómez-Duarte C, García VH, Manzi E, Escobar C, Jaramillo JD, Torres K, Millán M, Caicedo LA, Echeverri

GJ. Rev Colomb Cir. 2018;33:189-97. https://doi.org/10.30944/20117582.61 


\begin{abstract}
Background and objectives: Patients with brain death (BD) due to traumatic brain injury (TBI) secondary to a gunshot (GS) wound in the skull contribute to mitigate the demand for organ and tissue donors. In Colombia, $78 \%$ of the homicides are by GS. We describe our experience with BD secondary to TBI by GS organ and tissue donors at a transplant center in Cali, Colombia.

Materials and methods: This is a retrospective historical cohort study for characterization of donors diagnosed with BD secondary to TBI by GS at our institution in the period 2010-2016 ( $\mathrm{n}=\mathrm{I} 69)$. Qualitative variables were assessed by proportions, continuous quantitative variables with measures of central tendency, and survival with Kaplan Meier.

Results: The majority of the patients were male (93\%), with a median age of 2I years (RIQ 17-27), 32\% had history of psychoactive substance use. I $2 \%$ of the patient's required management with blood products and $62 \%$ with high doses of vasopressors. 338 solid organs were transplanted. Survival of the renal and hepatic graft at one year was $90 \%$ and $85 \%$ respectively and at five years of $87 \%$ and $76 \%$.

Conclusions: According to our study, the majority of these patients are young men with potentially transplantable organs, showing excellent results in the survival of the grafts. We consider these donors to be a good alternative to increase the number of organ and tissue donors.

Keywords: organ transplantation; donor selection; brain death; cerebrovascular trauma; brain injuries, traumatic; head injuries, penetrating.
\end{abstract}

\section{Introducción}

El trasplante de órganos puede constituir el único tratamiento curativo para pacientes con enfermedades crónicas avanzadas. No obstante, a nivel mundial el número de pacientes en lista de espera para trasplante aumenta exponencialmente, por lo cual se aumenta la brecha entre la demanda y el total de donantes de órganos y tejidos ${ }^{\mathrm{I}-4}$.

En el Reino Unido, en los años 20I4 y 2015, hubo un total de I.364 donantes efectivos de órganos, que permitieron hacer 3.519 trasplantes ${ }^{5}$. En Estados Unidos, según la United Network for Organ Sharing (UNOS) y la Organ Procurement and Transplantation Network(OPTN), hubo 24.986 trasplantes en el 2015 2,3. En Europa, según el Global Observatory on Donation and Transplantation (GODT), en el 2015 hubo un total de 3I.8I2 donantes efectivos, con un incremento de 5,58 \% con respecto al $2014^{6}$.

En Colombia, según las cifras del Instituto Nacional de Salud, en el año 2015 hubo un total de 405 donantes reales de órganos, con una tasa nacional de 8,4 donantes por millón de habitante, quienes fueron en su mayoría donantes cadavéri$\cos ^{7}$. La lista de espera del Instituto a finales del 2015, reportaba 2.029 pacientes que necesitaban órganos sólidos para trasplante, y se trasplantaron $\mathrm{I} .2 \mathrm{O} 4^{7}$.

Entre las estrategias para mitigar este déficit de donantes, se ha decidido caracterizar poblaciones emergentes de posibles donantes que previamente no se habían considerado como buenos candidatos para la donación al momento de fallecer. De estos, los casos de muerte encefálica por trauma craneoencefálico secundario a herida por proyectil de arma de fuego, pueden ser una alternativa viable.

En Colombia, durante el año 2015 se cometieron II. 585 homicidios $(24,03$ por cada IOo.00o habitantes), de los cuales el $23 \%$ fueron por trauma craneoencefálico $^{8}$. Según el análisis de situación de salud (ASIS), en el año 20I4, en el Valle del Cauca, la mortalidad por causa externa ocupó el cuarto lugar en general, de la cual, el 52,24\% de los casos correspondieron a homicidios (90,27 por cada I00.00o habitantes) ${ }^{9}$.

Existen pocos estudios que caractericen la población de donantes fallecidos por trauma craneoencefálico ${ }^{\mathrm{I}-\mathrm{I} 3}$. En Colombia, no existen datos recientes sobre la supervivencia de los injertos en los trasplantes de órganos y tejidos de donantes fallecidos por trauma craneoencefálico. 
En este estudio, se buscó describir la experiencia con donantes con muerte encefálica secundaria a trauma craneoencefálico por herida por proyectil de arma de fuego, en un centro latinoamericano de trasplantes.

\section{Materiales y métodos}

Se llevó a cabo un estudio de cohorte histórica retrospectiva, con las historias clínicas de los donantes cadavéricos de órganos y tejidos de la institución. Se incluyeron aquellos donantes efectivos con diagnóstico de muerte encefálica secundaria a trauma craneoencefálico por herida por proyectil de arma de fuego, en los que tanto la obtención de órganos como el trasplante de órganos sólidos abdominales se llevaron a cabo entre los años 2010 y 2016 en la Clínica Fundación Valle de Lili en Cali, Colombia. Se excluyeron aquellos donantes con diagnóstico de muerte encefálica secundaria a otras causas diferentes a trauma craneoencefálico, muerte circulatoria o muerte encefálica secundaria a tumores del sistema nervioso central.

Se incluyeron las variables sociodemográficas de edad, sexo, raza y escolaridad. Se consideró la gravedad del trauma: resultado de escala de Glasgow al ingreso, presión arterial sistólica, estado toxicológico, necesidad de vasopresores o hemoderivados, y el número de días transcurridos entre el ingreso del paciente a la institución y el diagnóstico de muerte encefálica. Asimismo, se recolectó información de los exámenes paraclínicos del donante. Además, se incluyeron las variables relevantes de la adquisición de órganos, tales como el tipo de líquido de preservación utilizado, el tiempo de la isquemia en frío y los órganos conseguidos. Finalmente, se analizaron la supervivencia del injerto y la del receptor.

Las variables categóricas se presentaron en proporciones y se analizaron para cada resultado de interés (pérdida del injerto, muerte o presencia de rechazo del injerto) con la prueba de ji al cuadrado. Se consideraron diferencias estadísticamente significativas aquellas con un valor de $\mathrm{p}$ menor de 0,05. Las variables continuas se expresaron como mediana con rango intercuartílico (RIQ) o promedios con desviación estándar (DE), dependiendo de la distribución de los datos, la cual fue evaluada con la prueba de Shapiro-Wilk. Se utilizaron pruebas no paramétricas (Mann-Withney o Wilcoxon) o paramétricas (t de Student o ANOVA) según la necesidad. La supervivencia del paciente y la del injerto se analizaron con el método de Kaplan-Meier. Todos los análisis se realizaron con Stata $\mathrm{I}^{\mathrm{TM}}$.

\section{Resultados}

Se obtuvo una serie de 169 pacientes con diagnóstico de muerte encefálica secundaria a trauma craneoencefálico por herida en cráneo por proyectil de arma de fuego del total de donantes $(n=470)$ que fueron donantes de órganos sólidos abdominales en la Clínica Fundación Valle de Lili entre 2010 y 2016 (figura I).

De los donantes, I58 (93\%) eran de sexo masculino y II3 (67\%) de raza mestiza, y la mediana de edad fue de 2I años (RIC=I7-27). En cuanto los datos relacionados a la escolaridad y ocupación, en una gran porción de los donantes no había registro ( $43 \%$ y $4 \mathrm{I} \%$, respectivamente); entre los datos registrados, el $36 \%$ de los casos tenía un nivel de estudio de secundaria y el I $_{5} \%$ estaba desempleado.

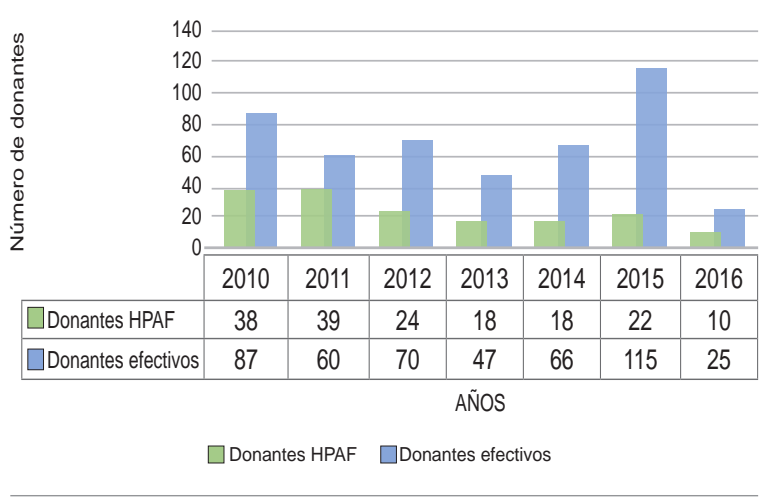

Figura 1. Comportamiento de los donantes con trauma craneoencefálico por herida por proyectil de arma de fuego de órganos sólidos, trasplantados en la Fundación Valle del Lili entre 2010 y 2016 
En cuanto a los datos del ingreso al servicio de urgencias, la mayoría (I3\%) de los donantes en quienes se registró el Glasgow, ingresaron con un puntaje de 3/15; sin embargo, en el $75 \%$ no hubo un registro adecuado de esta información. El 52,7 \% de los donantes fueron de grupo sanguíneo O, seguidos del grupo A (34\%). Se registró consumo de sustancias psicoactivas en $54(32 \%)$ y de alcohol en I6 (9,5\%) donantes. La presión arterial sistólica registrada al ingreso tuvo un promedio de IIO $\mathrm{mm} \mathrm{Hg}$ con desviación estándar de 19 (rango de 52 a I70). Se hizo el diagnóstico confirmatorio de muerte encefálica en menos de un día (I9 horas; $\mathrm{RIC}=0,46-\mathrm{I}, 08$ días) desde el ingreso.

Entre las ayudas diagnósticas empleadas en los donantes, se tomó muestra de sangre para exámenes paraclínicos al ingreso, la mayoría con resultados en rangos de normalidad (tabla I). Entre los resultados de las pruebas para infecciones, se encontró IgG positiva para citomegalovirus en I54 (9I \%) de los donantes.

En cuanto al soporte hemodinámico de los donantes, I44 (85\%) requirieron vasopresores y, de estos, IO5 (73\%) requirieron dosis de vasopresores consideradas altas, como noradrenalina $\geq 0,06 \mu \mathrm{g} / \mathrm{kg} / \mathrm{minuto}$, adrenalina $\geq 0,06 \mu \mathrm{g} / \mathrm{kg} / \mathrm{mi}-$ nuto, dopamina $\geq 4 \mu \mathrm{g} / \mathrm{kg} /$ minuto y vasopresina $\geq 2,5 \mathrm{U} /$ hora $^{\mathrm{I} 4-17}$. Se usó noradrenalina en $\mathrm{I} 36$ (94\%) de los donantes, con una mediana de dosis de $0,3 \mu \mathrm{g} / \mathrm{kg} / \mathrm{minuto}$ (RIC=0,I-0,625) durante cuatro horas ( $\mathrm{RIC}=\mathrm{I}, 75-\mathrm{IO})$. La combinación más común fue vasopresina con noradrenalina, en 47 (4I \%) de los donantes. Por otra parte, 2I (I2 \%) requirieron hemoderivados, con una mediana de 3,5 unidades de glóbulos rojos ( $\mathrm{RIC}=2-5$ ) (tabla 2).

De los I69 donantes efectivos de esta serie, se trasplantaron 338 órganos sólidos: I84 riñones, I25 hígados, 23 corazones, 8 páncreas, 4 pulmones, y uno de intestino (tabla 3). Además, se obtuvo un total de 8I tejidos oculares y 22 injertos de músculo esquelético, los cuales se destinaron a los bancos de ojos y hueso de la ciudad. La supervivencia del injerto renal y hepático a un año fue del $90 \%$ y $85 \%$, respectivamente, y a 5 años, del $87 \%$ y $76 \%$ (figura 2, figura 3 ).

\section{Discusión}

Conforme a los esfuerzos internacionales para disminuir el número de pacientes en lista de espera para trasplante de órganos ${ }^{18-20}$, como institución, hemos empleado varias estrategias educativas sobre la donación de órganos y tejidos, con el fin de sensibilizar a la comunidad, disminuir la tasa de negativas familiares a la donación y aumentar el número de donantes ${ }^{4-6,8, \mathrm{II}, 21-23}$.

Tabla 1. Resultados de los exámenes de laboratorio de los donantes

\begin{tabular}{|c|c|}
\hline Característica & Valor $(n=169)$ \\
\hline $\begin{array}{l}\text { Creatinina sérica }(\mathrm{mg} / \mathrm{dl}) \\
\text { Mediana }(\mathrm{RIC}) \\
\text { Rango }\end{array}$ & $\begin{array}{c}1 \\
(0,83-1,3) \\
0,37-3,46\end{array}$ \\
\hline $\begin{array}{l}\text { Hemoglobina }(\mathrm{g} / \mathrm{dl}) \\
\text { Promedio } \pm \mathrm{DE} \\
\text { Rango }\end{array}$ & $\begin{array}{l}10 \pm 2,6 \\
1,2-18\end{array}$ \\
\hline $\begin{array}{l}\text { Plaquetas }\left(\mathrm{mm} \text { por } 10^{3}\right) \\
\text { Mediana (RIC) } \\
\text { Rango }\end{array}$ & $\begin{array}{c}144 \\
(107,5-188,25) \\
10-462\end{array}$ \\
\hline $\begin{array}{l}\text { Tiempo de protombina (s) } \\
\text { Mediana (RIC) } \\
\text { Rango }\end{array}$ & $\begin{array}{c}17,3 \\
(13-22) \\
8,2-180\end{array}$ \\
\hline $\begin{array}{l}\text { Tiempo de tromboplastina (s) } \\
\text { Medina (RIC) } \\
\text { Rango }\end{array}$ & $\begin{array}{c}35 \\
(29-43) \\
11-180\end{array}$ \\
\hline $\begin{array}{l}\text { INR } \\
\text { Mediana (RIC) } \\
\text { Rango }\end{array}$ & $\begin{array}{c}1,4 \\
(1,2-1,8) \\
0,83-6\end{array}$ \\
\hline $\begin{array}{l}\text { Sodio sérico (mEq/L) } \\
\text { Mediana (RIC) } \\
\text { Rango }\end{array}$ & $\begin{array}{c}144(141-153) \\
132-182\end{array}$ \\
\hline $\begin{array}{l}\text { Potasio sérico }(\mathrm{mEq} / \mathrm{L}) \\
\text { Promedio } \pm \mathrm{DE} \\
\text { Rango }\end{array}$ & $\begin{array}{c}4,25 \pm 1,16 \\
1,44-7,39\end{array}$ \\
\hline $\begin{array}{l}\text { ALT (UI/L) } \\
\text { Mediana (RIC) } \\
\text { Rango }\end{array}$ & $\begin{array}{c}26 \\
(15-42) \\
3,6-297\end{array}$ \\
\hline $\begin{array}{l}\text { AST (UI/L) } \\
\text { Mediana (RIC) } \\
\text { Rango }\end{array}$ & $\begin{array}{l}55(38-81) \\
10,6-424,9\end{array}$ \\
\hline $\begin{array}{l}\text { Fosfatasa alcalina (UI/L) } \\
\text { Mediana (RIC) } \\
\text { Rango }\end{array}$ & $\begin{array}{c}69 \\
(47-91) \\
7,6-333\end{array}$ \\
\hline $\begin{array}{l}\text { Bilirrubina (mg/L) } \\
\text { Mediana (RIC) } \\
\text { Rango }\end{array}$ & $\begin{array}{c}0,75 \\
(0,43-1,09) \\
0,1-3,6\end{array}$ \\
\hline
\end{tabular}


Tabla 2. Uso de vasopresores

\begin{tabular}{|c|c|c|c|}
\hline $\begin{array}{c}\text { Vasopresor } \\
(\mathrm{N}=144)\end{array}$ & n (\%) & Dosis & $\begin{array}{l}\text { Tiempo } \\
\text { (horas) }\end{array}$ \\
\hline $\begin{array}{l}\text { Dopamina } \\
\text { ( } \mu \mathrm{g} / \mathrm{kg} / \text { minuto) }\end{array}$ & $16(11)$ & 8 & 6,5 \\
\hline Mediana (RIC) & & $(5-14)$ & $(2-9)$ \\
\hline Rango & & $0,2-20$ & $1-30$ \\
\hline $\begin{array}{l}\text { Vasopresina } \\
\text { (U/kg/minuto) }\end{array}$ & $49(34)$ & & \\
\hline Mediana (RIC) & & $2(2-5)$ & $8(2,5-12)$ \\
\hline Rango & & $0,01-50$ & $1-24$ \\
\hline $\begin{array}{l}\text { Adrenalina } \\
\text { ( } \mu \mathrm{g} / \mathrm{kg} / \text { minuto) }\end{array}$ & $4(2.8)$ & & \\
\hline Mediana (RIC) & & $1(0,47-1,45)$ & $2,3(0,5-6,25)$ \\
\hline Rango & & $0,3-1,6$ & $0,5-7$ \\
\hline $\begin{array}{l}\text { Noradrenalina } \\
(\mu \mathrm{g} / \mathrm{kg} / \mathrm{minuto})\end{array}$ & $136(94)$ & & \\
\hline Mediana (RIC) & & $0,3(0,1-0,625)$ & $4(1,75-10)$ \\
\hline Rango & & $0,02-6$ & $0,004-48$ \\
\hline $\begin{array}{l}\text { Vasopresina y } \\
\text { noradrenalina } \\
\text { Vasopresina }\end{array}$ & $47(41)$ & & \\
\hline Mediana (RIC) & & $2(2-5)$ & $8(2-12)$ \\
\hline Rango & & $0,01-50$ & 1-24 \\
\hline \multicolumn{4}{|l|}{ Noradrenalina } \\
\hline Mediana (RIC) & & $0,5(0,1-1)$ & $4(1-10)$ \\
\hline Rango & & 0,04-4 & $0,004-40$ \\
\hline
\end{tabular}

Tabla 3. Órganos donados y trasplantados en Fundación Valle del Lili

\begin{tabular}{lrc}
\hline \multicolumn{1}{c}{ Órganos donados } & $\mathbf{n}$ & (\%) \\
\hline Hígado & 125 & 74 \\
Riñón & 184 & 54 \\
Corazón & 23 & 13,6 \\
Páncreas & 8 & 4,7 \\
Válvulas cardiacas & 7 & 4,14 \\
Pulmón & 4 & 2,3 \\
Intestino & 1 & 0,6 \\
Tráquea y laringe & 1 & 0,6 \\
\hline
\end{tabular}

Asimismo, a nivel regional, la Universidad ICESI en convenio con la Fundación Valle de Lili ha implementado el diplomado en coordinación operativa de trasplantes, el cual busca formar profesionales expertos en la promoción, la donación, el proceso de detección, el mantenimiento del donante y los aspectos logísticos relacionados con la donación.
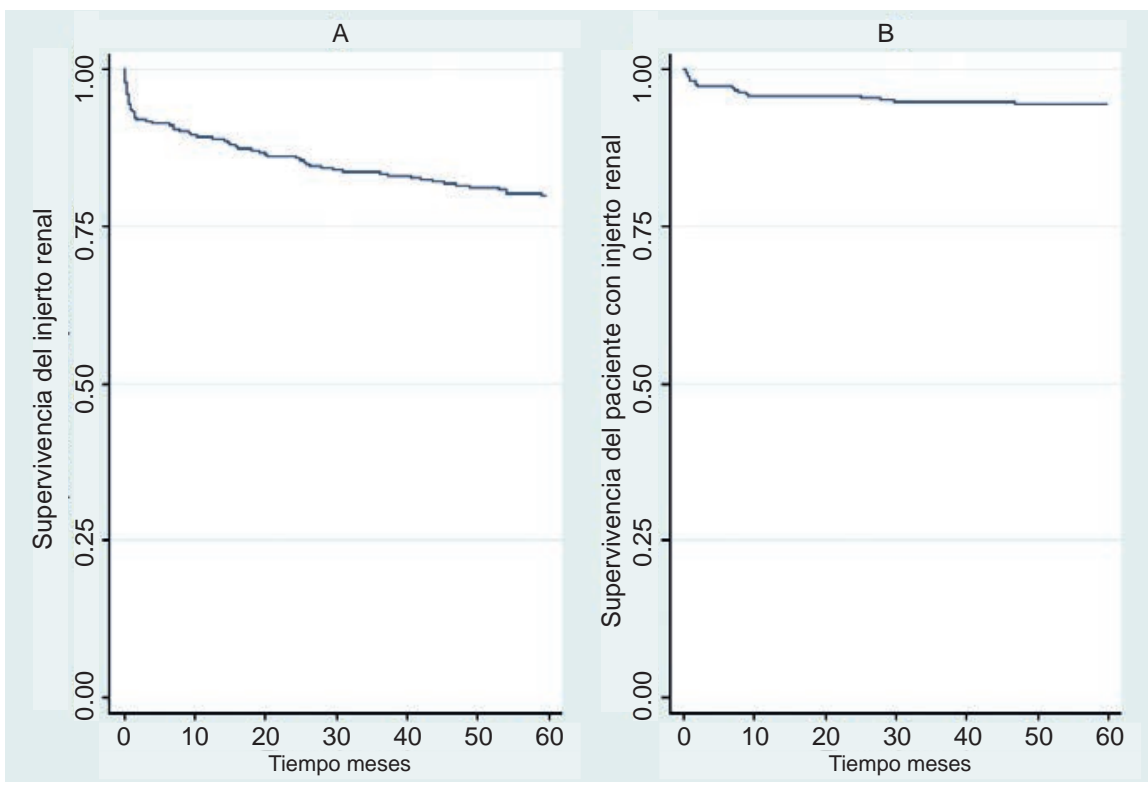

Figura 2. Tiempo de supervivencia del injerto renal y paciente receptores del injerto proveniente de donante con muerte cerebral por trauma craneoencefálico debido a herida por proyectil de arma de fuego 


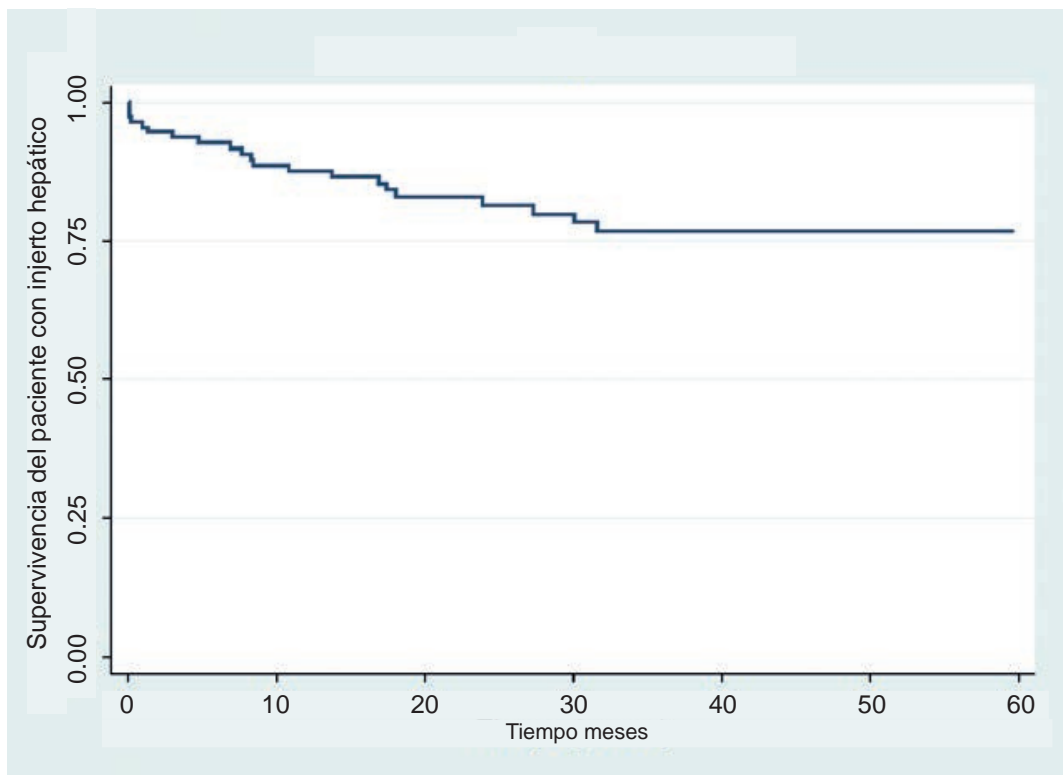

Figura 3. Tiempo de supervivencia del injerto hepático y paciente receptores del injerto proveniente de donante con muerte cerebral por trauma craneoencefálico debido a herida por proyectil de arma de fuego

Además, a nivel nacional, el Congreso de la República expidió la Ley 1805 de 20I6, por medio de la cual se modifican la Ley 73 de 1988 y la Ley 919 de 2004, ampliando la presunción legal de componentes anatómicos para fines de trasplantes u otros usos terapéuticos. Esta ley pretende brindar mayores oportunidades para aquellas personas que padecen enfermedades crónicas terminales, atribuyendo la facultad de oponerse a la donación de componentes anatómicos, únicamente, a la persona que en vida así lo hubiera manifestado formalmente. Además, exige a las Instituciones Prestadoras de Servicios de Salud contar con la capacidad técnica necesaria para detectar, en tiempo real, a los potenciales donantes, de acuerdo con los criterios y competencias que establezca el Instituto Nacional de Salud.

Se estima que de 3I a 35,5\% de los donantes de órganos en Estados Unidos provienen de muertes por trauma ${ }^{13,24}$, cifra que duplica a las donaciones provenientes de enfermedades no traumáticas. En Holanda se reportó que, de un total de I04 pacientes que ingresaron por trauma craneoencefálico, I5 (I4 \%) fueron donantes efectivos de órganos; no obstante, no se registraron heridas por proyectil de arma de fuego ${ }^{13}$. En Estados Unidos, en un estudio de 135 pacientes, reportaron que hasta el $24 \%$ de los donantes murieron por herida por proyectil de arma de fuego ${ }^{25}$. En Europa, el 7,8 \% de los donantes fallecen por muerte encefálica; en España, 2,3\% mueren por muerte encefálica y, en estos, los accidentes de tránsito son la principal causa; sin embargo, no se reportaron cifras de herida por proyectil de arma de fuego ${ }^{26}$. Además, según Bodi, et al., de I39 pacientes admitidos con diagnóstico de trauma craneoencefálico en nueve centros de España, 27 progresaron a muerte encefálica y 2I fueron donantes efectivos, sin discriminar la etiología del trauma craneoencefálico (trauma cerrado o por herida con proyectil de arma de fuego) ${ }^{22}$.

Siendo Colombia un país en donde la violencia es una de las principales causas de muerte ${ }^{8,9}$, hemos considerado una estrategia exitosa considerar donantes con muerte encefálica por herida con arma de fuego, para aumentar el número de donantes y disminuir las listas de espera de órganos. 
Este tipo de trauma craneoencefálico es muy frecuente en nuestro medio ${ }^{8}$. Según la literatura internacional, la mortalidad en el lugar de los hechos es del 7I \% y, a los 3 meses, de 70 a 90 $\%$ según el trayecto del proyectil y el puntaje de Glasgow al ingreso ${ }^{12,26-28}$. En el $75 \%$ de los casos, el diagnóstico de muerte encefálica es temprano (menos de un día), pues, cuando el paciente ingresa a urgencias, el trauma es muy grave y el puntaje de Glasgow es de 3/15 o menor. Por lo tanto, es necesario un tratamiento óptimo y rápido, con el fin de mantener la viabilidad de los órganos o tejidos, para que sean aptos para trasplante ${ }^{12,13,26,27,29}$.

Durante el periodo de seis años de estudio (20I0-20I6) en nuestra institución, se obtuvo un total de 470 donantes efectivos, de los cuales I69 (36\%) tenían diagnóstico de muerte encefálica por trauma craneoencefálico secundario a herida por proyectil de arma de fuego en el cráneo. Esto concuerda con los reportes internacionales que muestran proporciones similares de donantes con estas mismas características ( $15 \%, 35 \%$ y $49 \%)^{\mathrm{I}, \mathrm{I}, 29}$. El aumento de estos porcentajes se ha visto estrechamente relacionado con la existencia de protocolos de atención para los pacientes con muerte encefálica, los avances en la prevención de accidentes de tránsito y las mejoras en la atención del paciente neurológico crítico, lo cual ha mejorado las tasas de obtención de órganos hasta en 7I \% ${ }^{15,16}$. Lo anterior coincide con la presencia de protocolos estandarizados para el mantenimiento del estado hemodinámico de los donantes en nuestra institución ${ }^{30}$.

En la presente serie, el $93 \%$ de los donantes era de sexo masculino, con una mediana de edad de 2I años; entre los antecedentes personales, aproximadamente, un tercio de la población analizada tenía consumo de algún tóxico, siendo las sustancias psicoactivas las más frecuentes. En la literatura científica no hay reporte de una cohorte de donantes con muerte encefálica por trauma craneoencefálico secundario a herida por arma de fuego tan grande como esta ${ }^{\mathrm{I}, \mathrm{I}, 29}$. No obstante, las características sociodemográficas son similares, y hay coincidencia en el predominio del sexo masculino en todas las series, con frecuencias mayores al $80 \%^{1,13,25,31}$. La edad promedio que se reporta en la población de Estados Unidos es de 38 años, edad que casi duplica la de nuestra población ${ }^{9,15}$.

El evitar la inestabilidad hemodinámica en estos pacientes es fundamental para la preservación de la función del órgano después de la muerte encefálica ${ }^{55,17}$. En el presente estudio, la mayoría de los pacientes tenía una presión arterial sistólica promedio cercana a lo reportado y el $85 \%$ requirió vasopresores a dosis altas, $\mathrm{I} \mu \mathrm{g} /$ $\mathrm{kg} /$ minuto de adrenalina ( $\mathrm{RIC}=0,47-\mathrm{I}, 45), 0,3 \mu \mathrm{g} /$ $\mathrm{kg} /$ minuto de noradrenalina $(\mathrm{RIC}=\mathrm{O}, \mathrm{I}-\mathrm{0}, 625)$ o 2 $\mathrm{U} / \mathrm{kg} /$ minuto de vasopresina $(\mathrm{RIC}=2-5$ ), por periodos prolongados y en combinación (tabla 2). Esta definición de dosis altas de catecolaminas sigue siendo un tema de controversia entre diferentes autores ${ }^{17,32,33}$. La meta para el mantenimiento del donante es clara y hace necesario mantener una presión arterial media entre 65 y $90 \mathrm{~mm} \mathrm{Hg}$, con un adecuado soporte de líquidos intravenosos $y$, de ser necesario, fármacos vasoactivos ${ }^{17,30}$. La vasopresina está indicada como tratamiento de la diabetes insípida, para mejorar la contractibilidad miocárdica y el gasto urinario, y para disminuir la dosis necesaria de catecolaminas en el mantenimiento del donante $1,17,25,34$.

El número de injertos hepáticos, renales e intestinales procurados en nuestra institución, es similar al de otros centros a nivel internacional ${ }^{1,25}$. No obstante, en realidad, el número de injertos renales y hepáticos obtenidos fue mayor, pero algunos fueron trasladados a otras instituciones para su trasplante, por lo que no se tuvieron en cuenta en este estudio pues no se podía hacer el seguimiento. El número de injertos cardiacos, pancreáticos y de pulmón es menor en comparación con cifras internacionales; no obstante, a nivel regional somos pioneros en estos procedimientos.

Los pacientes que presentan muerte encefálica por trauma craneoencefálico secundario a herida de arma de fuego en cráneo, son jóvenes y no tienen antecedente de enfermedades asociadas, lo que los convierte en excelentes candidatos a la donación. Además, al analizar la supervivencia 
de los injertos de estos donantes, se encontró que el injerto renal tuvo una supervivencia del 9o \% al año y de $85 \%$ a los cinco años, y el injerto hepático, de $87 \%$ al año y de $76 \%$ a los cinco años. Esto es comparable con lo reportado en Europa, con cifras de 90 a $92 \%$ al año y $75 \%$ a los cinco años para el injerto renal ${ }^{35,36}$, y del $86 \%$ al año y el $80 \%$ a los cinco años para el injerto hepático ${ }^{37}$.

Entre las limitaciones del presente estudio, la muestra de la población es de un solo centro de una ciudad de Colombia. Por otra parte, la recolección de los datos fue retrospectiva y se encontró un déficit importante en el registro de algunos de los datos de los donantes, lo cual fue mejorando al pasar de los años.

\section{Conclusiones}

Según el presente estudio, la mayoría de estos pacientes son hombres jóvenes, con órganos potencialmente trasplantables. El análisis de supervivencia mostró resultados satisfactorios, con altos porcentajes de supervivencia de los injertos renal y hepático a uno y cinco años. Lo anterior fortalece nuestra decisión de usar donantes con muerte encefálica por trauma craneoencefálico secundario a herida por proyectil de arma de fuego en cráneo, demostrando que son excelentes candidatos a la donación. Esta es una estrategia útil para aumentar el número de donantes de órganos y tejidos. No obstante, es necesario hacer más estudios con el fin de caracterizar y estandarizar el manejo de este tipo de pacientes, ya que no existe mucha información al respecto.

\section{Conflicto de intereses}

No existe ninguno.

\section{Financiamiento}

No se recibió financiación alguna para llevar a cabo el presente estudio.

\section{Referencias}

I. Croezen DH, van Natta TL. Presentation and outcomes for organ donation in patients with cerebral gunshot wounds. Clin Transplant. 200I;I5(Suppl.6):II-5.

2. Organ Procurement and Transplantation Network. Fecha de consulta: 9 de mayo de 20I7. Disponible en: https:/optn.transplant.hrsa.gov/data/.
3. Data | UNOS. Fecha de consulta: 9 de mayo de 2017. Disponible en: https://www.unos.org/data/.

4. Sung RS, Galloway J, Tuttle-Newhall JE, Mone T, Laeng $\mathrm{R}$, Freise CE, et al. Organ donation and utilization in the United States, 1997-2006. Am J Transplant. 2008;8:92234. https://doi.org/I0.IIII/j.I60o-6I43.2008.02I7I.X

5. Johnson S, Forsythe J, Murphy P. Organ donation and transplantation activity report 2015/I6. Fecha de consulta: 9 de mayo de 20I7. Disponible en: nhsbtdbe. blob.core.windows.net/umbraco-assets-corp/I452/activity_report_20I5_I6.pdf.

6. Organ donation and transplantation activities. Godt. 20I7. Fecha de consulta: Io de enero de 20I8. Disponible en: http://www.transplant-observatory.org/.

7. Instituto Nacional de Salud, Coordinación Nacional Red Donación y Trasplantes. Informe anual Red de Donación y Trasplantes Colombia, año 2015. Fecha de consulta: 9 de mayo de 20I7. Disponible en: http:// www.ins.gov.co/lineas-de-accion/Red-Nacional-Laboratorios/Estadsticas/Informe\%2oRed\%20de\%2oDonaci\%C3\%B3n\%20y\%20Trasplante\%202015.pdf.

8. Grupo Centro de Referencia Nacional sobre Violencia. Instituto Nacional de Medicina Legal y Ciencias Forenses. Comportamiento del homicidio. Colombia 20I5. Fecha de consulta: 9 de mayo de 2017. Disponible en: http://www.medicinalegal.gov.co/documents/88730/34I8907/2.+homicidios.pdf/70a4c34b920c-465b-9902-936ffeab4afd.

9. Secretaría Departamental del Valle del Cauca, Grupo de Salud Pública, Grupo ASIS. Análisis de situación de salud con el modelo de los determinantes sociales Valle del Cauca año 20I6. Fecha de consulta: Io de mayo de 20I7. Disponible en: www.valledelcauca.gov.co/salud/ descargar.php?id=6288.

Io. Brody A, Kashuk JL, Moore EE, Beauchamp K, Barnett C, Biffl WL, et al. Fatal gunshot wounds to the head: A critical appraisal of organ donation rates. Am J Surg. 2010;200:728-33.

II. Freitas RA de, Dell'Agnolo CM, Alves EF, Benguella EA, Pelloso SM, Carvalho MDB. Organ and tissue donation for transplantation from fatal trauma victims. Transplant Proc. 2015;47:874-8.

I2. Alarhayem AQ, Cohn SM, Muir MT, Myers JG, Fuqua J, Eastridge BJ. Organ donation, an unexpected benefit of aggressive resuscitation of trauma patients presenting dead on arrival. J Am Coll Surg. 20I7;224:926-32.

I3. Kompanje EJO, Bakker J, Slieker FJA, Ijzermans JNM, Maas AIR. Organ donations and unused potential donations in traumatic brain injury, subarachnoid haemorrhage and intracerebral haemorrhage. Intensive Care Med. 2006;32:217-22.

I4. Herget-Rosenthal S, Saner F, Chawla LS. Approach to hemodynamic shock and vasopressors. Clin J Am Soc Nephrol. 2008;3:546-53. 
I5. Kotloff RM, Blosser S, Fulda GJ, Malinoski D, Ahya $\mathrm{VN}$, Angel L, et al. Management of the potential organ donor in the ICU: Society of Critical Care Medicine/ American College of Chest Physicians/Association of Organ Procurement Organizations Consensus Statement. Crit Care Med. 2015;43:129I-325.

I6. Rungta N, Pande R, Munjal M, Khunteta S. Critical Care. London: JP Medical Ltd.; 20I5. p. 674.

I7. Chamorro-Jambrina C, Muñoz-Ramírez MR, Martínez-Melgar JL, Pérez-Cornejo MS. Organ donor management: Eight common recommendations and actions that deserve reflection. Med Intensiva. 20I7;4I:559-68.

I8. Matesanz R. The diagnosis of brain death in Latin America. Med Intensiva. 2009;33:413-4.

19. García A, Gómez MP. Organizational model for a national system of donation and transplantation from deceased donors in Nicaragua. Transplant Proc. 2OII;43:331O-I.

20. Escudero D, Matesanz R, Soratti CA, Flores JI, Red/ Consejo Iberoamericano de Donación y Trasplante. Brain death in Ibero-America. Med Intensiva. 2009;33:4I5-23.

2I. Torres K, Aristizábal AM, Echeverri GJ, Cuenca S, Roldán M, Escobar V, et al. "Hospital donante" una estrategia educativa: experiencia de un centro latinoamericano de trasplantes. Rev Colomb Cir. 20I7;32:205I3. https://doi.org/IO.30944/20117582.26.

22. Bodí MA, Pont T, Sandiumenge A, Oliver E, Gener J, Badía $\mathrm{M}$, et al. Potencialidad de donación de órganos en muerte encefálica y limitación del tratamiento de soporte vital en los pacientes neurocríticos. Med Intensiva. 2015;39:337-44.

23. Molina MI, Toro PA, Manzi E, Davalos D, Aristizábal AM, Gómez C, et al. Principales causas de negativa familiar a la donación de órganos y tejidos: Io años de experiencia en un centro latinoamericano. Rev Soc Esp Nefrol. 20I7; En prensa.

24. Raoof M, Joseph BA, Friese RS, Kulvatunyou N, O'Keeffe T, Tang A, et al. Organ donation after traumatic cardiopulmonary arrest. Am J Surg. 20II;202:70I-5.

25. Kemp CD, Cotton BA, Johnson JC, Ellzey M, Pinson CW. Donor conversion and organ yield in traumatic brain injury patients: Missed opportunities and missed organs. J Trauma. 2008;64:1573-80.
26. Escudero D, Otero J. Medicina intensiva y donación de órganos. ¿Explorando las últimas fronteras? Med Intensiva. 2015;39:366-74.

27. Aras M, Altaş M, Yilmaz A, Serarslan Y, Yilmaz N, Yengil $\mathrm{E}$, et al. Being a neighbor to Syria: A retrospective analysis of patients brought to our clinic for cranial gunshot wounds in the Syrian civil war. Clin Neurol Neurosurg. 20I4;125:222-8.

28. Aarabi B, Tofighi B, Kufera JA, Hadley J, Ahn ES, Cooper C, et al. Predictors of outcome in civilian gunshot wounds to the head. J Neurosurg. 2014;120:I138-46.

29. Joseph B, Aziz H, Sadoun M, Kulvatunyou N, Pandit V, Tang A, et al. Fatal gunshot wound to the head: The impact of aggressive management. Am J Surg. 2014;207:8994 .

30. Aristizábal AM, Castrillón Y, Gil T, Restrepo D, Solano $\mathrm{K}$, Guevara M, et al. Manejo actual del donante potencial de órganos y tejidos en muerte cerebral: guía de manejo y revisión de la literatura. Rev Colomb Cir. 20I7;32:I28-45. https://doi.org/I0.30944/20II7582.I7

31. Alted E, Bermejo S, Chico M. Actualizaciones en el manejo del traumatismo craneoencefálico grave. Med Intensiva. 2009;33:16-30.

32. Citerio G, Cypel M, Dobb GJ, Domínguez-Gil B, Frontera JA, Greer DM, et al. Organ donation in adults: A critical care perspective. Intensive Care Med. 2016;42:305-I5.

33. DuBose J, Salim A. Aggressive organ donor management protocol. J Intensive Care Med. 2008;23:367-75.

34. Rostron AJ, Avlonitis VS, Kirby JA, Dark JH. Hemodynamic resuscitation of the brain-dead organ donor and the potential role of vasopressin. Transplant Rev. 2007;21:34-42.

35. Moreso F, Hernández D. ¿Ha mejorado la supervivencia del injerto tras el trasplante renal en la era de la moderna inmunosupresión? Revista Nefrología. 2013;33:14-26.

36. CTS-Collaborative Transplant Study. Fecha de consulta: 30 de junio de 20I7. Disponible en: http://www. ctstransplant.org/public/introduction.shtml

37. Bellingham JM, Santhanakrishnan C, Neidlinger N, Wai P, Kim J, Niederhaus S, et al. Donation after cardiac death: A 29-year experience. Surgery. 20II;I50:692-702. 